\title{
Ingenol mebutate treatment in actinic keratosis - clinical effectiveness and potential side effects
}

\author{
Aleksandra Lesiak ${ }^{1}$ Anna Maćkowska², Igor A. Bednarski', Paweł Kolano ${ }^{3}$, Irmina Olejniczak-Staruch ${ }^{4}$, \\ Anna Woźniacka ${ }^{4}$, Joanna Sieniawska ${ }^{4}$, Joanna Narbutt ${ }^{1}$
}

${ }^{1}$ Department of Dermatology, Pediatric Dermatology and Oncology, Medical University of Lodz, Lodz, Poland ${ }^{2}$ Student Research Group at Department of Dermatology, Pediatric Dermatology and Oncology, Medical University of Lodz, Lodz, Poland ${ }^{3}$ Department of General and Oncological Surgery, Tomaszow Health Centre, Tomaszow Mazowiecki, Poland ${ }^{4}$ Department of Dermatology and Venereology, Medical University of Lodz, Lodz, Poland

Adv Dermatol Allergol 2019; XXXVI (4): 468-471

DOI: https://doi.org/10.5114/ada.2019.87450

\begin{abstract}
Introduction: Actinic keratosis is a common skin disease that occurs in response to prolonged exposure to ultraviolet radiation. This problem affects up to $60 \%$ of the population over 40 years of age. Actinic keratosis is considered to be a precancerous lesion leading to squamous cell carcinoma (SCC). The new therapeutic option for the treatment of actinic keratosis is ingenol mebutate gel (0.015\%, 0.05\%).

Aim: Retrospective evaluation of response and potential side effects of ingenol mebutate treatment in clinical practice.

Material and methods: Eight patients with actinic keratosis lesions on the face or scalp self-applied a $0.015 \%$ gel for 3 consecutive days on the $25 \mathrm{~cm}^{2}$ marked area. They were assessed at baseline and on day 4, 7, 14 and 57 .

Results: All patients on day 57 presented a complete absence of AK lesions in the area of ingenol mebutate application. No adverse events were observed.

Conclusions: Our study shows that ingenol mebutate is highly efficacious field treatment for actinic keratosis.

Key words: actinic keratosis, ingenol mebutate.
\end{abstract}

\section{Introduction}

Actinic keratosis (AK) is a common skin disease that occurs in response to excessive exposure to ultraviolet (UV) radiation. This disease affects up to $60 \%$ of general population over 40 years of age, that is highly exposed to ultraviolet radiation [1]. Prevalence is dependent on many factors such as age, gender, latitude, fair skin (Fitzpatrick skin types I-III), but also immunodeficiencies or human papillomavirus infections [2].

Actinic keratosis is a disease characterized by the presence of hyperkeratotic, scaly papules or plaques, limited to the epidermis. Changes occur more often in places exposed to the ultraviolet radiation than other, e.g. the face, scalp and forearms. Actinic keratosis is considered to be a precancerous lesion that, in some cases, might lead to squamous cell carcinoma (SCC) [3]. Although the probability of a tumor transformation of singular lesions is quite low, it should be noted that patients who were highly exposed to the ultraviolet radiation have many AK changes, which is why the overall risk of squamous cell carcinoma is cumulative. It is estimated that a patient with 7-8 lesions of AK has a 6.1-10.2\% risk of developing SCC within 10 years. In addition, $60-82 \%$ of SCCS are produced directly at or near the AK changes [4].

Unfortunately, it is not possible to assess which AK plaques will transform into SCC, thus it is important to treat AK lesions effectively.

Treatment regimens in AK could be divided into lesion-directed and field-directed therapies. Selecting a therapy for a particular patient must always be supported by a history of disease, the amount and localization of lesions, the overall level of sun damage, and the patient's ability to cooperate and willingness to follow medical advice. Lesion-directed therapy consists of cryotherapy, curettage with electrostimulation and chemical peels. Cryosurgery with liquid nitrogen is one of the most commonly used methods in the US [5] and is dedicated to people with many, not very large and well-demarcated

Address for correspondence: Igor A. Bednarski MD, Department of Dermatology, Pediatric Dermatology and Oncology, Medical University of Lodz, 1/5 Kniaziewicza St, 91-347 Lodz, Poland, phone: + 48666379 108,

e-mail: igorbednarskiv@gmail.com

Received: 5.03.2018, accepted: 11.05.2018. 
changes. Unfortunately, the common side effect of this therapy is hypopigmentation of the skin as well as pain during the treatment. In addition, changes tend to recur [6]. The field-directed therapy is targeted at people with multiple lesions located in similar localizations and with a subclinical AK. This approach includes methods such as dermabrasion, photodynamic and laser therapy, as well as topical agents: 5-fluorouracil, imiquimod, 3\% diclofenac gel. A large limitation of topical application is their recommended time of use. Imiquimod, depending on the formulation, must be used for weeks/months, fluorouracil for weeks, and diclofenac for months [7]. In addition, according to the literature, 5-fluorouracil and imiquimod cause skin irritation that leads to a serious discomfort, intolerable to a number of patients. It results in discontinuation of the treatment. On the other hand, diclofenac can cause allergic reactions [8].

The best results in treating AK lesions have been achieved by combining lesion-directed and field-directed techniques. It is reported that combining diclofenac [9] with imiquimod [10] or 5-fluorouracil [11], before cryotherapy, is successful.

The new therapeutic option for the treatment of actinic keratosis is ingenol mebutate gel (0.015\%, 0.05\%). A $0.015 \%$ gel is intended for daily use on the face or scalp for 3 consecutive days, while a $0.05 \%$ gel is dedicated to patients with AK lesions in the extremities and trunk and should be applied once a day for 2 consecutive days. In our article we would like to present results of the treatment of 10 patients in the Department of Dermatology and Venereology of the Medical University of Lodz.

\section{Aim}

Retrospective evaluation of response and potential side effects of ingenol mebutate treatment in the clinical practice among Polish patients.
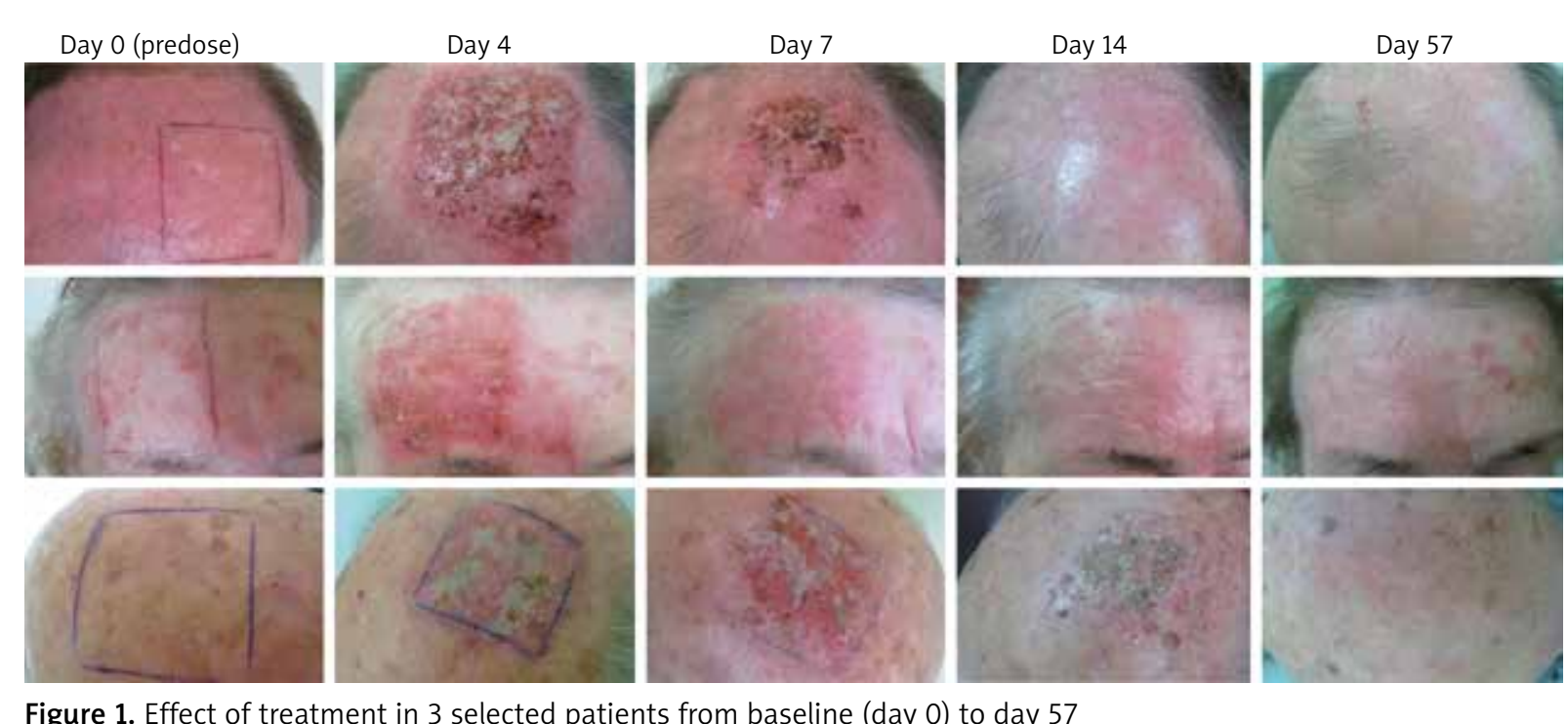

Figure 1. Effect of treatment in 3 selected patients from baseline (day 0 ) to day 57

\section{Material and methods}

ten patients of the Department of Dermatology and Venereology of the Medical University of Lodz were qualified for ingenol mebutate treatment. All patients presented Fitzpatrick phototype I, II or III and had changes of actinic keratosis within the scalp (scalp or forehead). Patients were aged between 68 and 84 years. The study involved 9 men and 1 woman. Each patient presented an area of $25 \mathrm{~cm}^{2}$ occupied by AK changes. Patients with hypertrophic lesions, who recently used immunosuppressive or immunomodulatory medications, were excluded. Patients had been self-applying a $0.015 \%$ gel on the marked area for 3 consecutive days. Each of them was assessed at baseline and on day 4, 7, 14 and 57.

The effects of treatment are shown in Figure 1.

On day 57, all patients presented a complete absence of AK lesions in the area of ingenol mebutate application. In every treated patient there were observable local skin reactions that could be classified as erythema, flaking, crusting and swelling, of various intensity. The presence and intensification of those local reactions peaked on day 4. Two patients presented a severe local skin reaction with a bacterial superinfection. They received antibiotics: doxycycline (200 mg in two doses for 10 days) and fusidic acid (applied on the skin twice a day for 10 days). Four patients had moderate and other 4 mild local skin reactions. However, those symptoms were temporary and not troublesome for the patients.

\section{Discussion}

Ingenol mebutate is a diterpene ester, which is extracted from the plant called Euphorbia peplus. In the past, the sap of the plant was used as a remedy for many skin diseases [12].

Ingenol mebutate has multiple mechanisms of action. One of them is an initial chemoablation by disrup- 
tion of the plasma membrane and mitochondria, which leads to a loss of mitochondrial membrane potential and, ultimately, necrosis of locally affected cells. The second mechanism of action is elimination of tumor cells by the tumor-specific antibodies, which proinflammatory cytokines and massive releasing of neutrophils - leading to neutrophil-and antibody dependent cellular cytotoxicity $[13,14]$.

Lebwohl et al. [7] in two of their studies confirmed a high efficacy of ingenol mebutate. Studies $(n=547)$ examined the ingenol mebutate $0.015 \%$ gel versus a vehicle for 3 days in AK on the face or scalp while other studies $(n=458)$ tested ingenol mebutate $0.05 \%$ versus a vehicle for 2 days in AK on the trunk or extremities. Patients had been applying the substance on AK areas of $25 \mathrm{~cm}^{2}$. The median reduction in the lesion count was $83 \%$ with $0.015 \%$ gel and $75 \%$ with $0.05 \%$ gel, compared to a $0 \%$ reduction in both groups using the vehicle. Among patients using ingenol mebutate on the scalp, the most common skin reaction was pain (13.9\%), pruritus (8\%) and irritation (1.8\%). No serious adverse events were observed [7]. Moreover, Jim On et al. [15] analyzed a relation between AK lesions count at week 8 adjusted for baseline and composite LSR score (local skin reaction score). The percentage reduction in AK lesions was higher in patients with a higher LSR score. A large skin reaction after ingenol mebutate treatment gives more reliable probability of AK clearance [15].

The same clearance rate as after using ingenol mebutate for 2-3 days is achieved with other field therapies but it requires a longer treatment period. Using 5-fluorouracil in a 4-week treatment leads to a complete clearance rate of $43 \%$ [16]. A treatment with imiquimod causes complete clearance rates from $25 \%$ to $35.5 \%$ after 2 or 3 weeks' treatment [17].

In another trial, patients received ingenol mebutate on approximately $250 \mathrm{~cm}^{2}$ sun-damaged skin for three consecutive days. Of 61 patients, 10 had a subnanomolar level of ingenol mebutate in whole blood (0.235-0.462 nM). No serious adverse reactions were observed, most of them were mild to moderate in intensity [18].

Ingenol mebutate could also be a concurrent treatment used in order to increase the effectiveness of the therapy. Hashim et al. confirm that application of the $0.05 \%$ ingenol mebutate gel on the same day as a cryosurgery is more effective in reducing numerous hyperkeratotic actinic keratosis lesions ( -4.3 vs. -2.8 in the control group) and non-hyperkeratotic lesions ( -3.8 vs. -0.8 in the control group). No significant increase in the number of local skin reactions was observed [19].

Erlendsson et al. demonstrated in a trial with hairless mice that ingenol mebutate prevents progression of UV-induced photodamages. Sixty hairless mice were exposed to the ultraviolet radiation and received five single treatments at 4 weeks' intervals. On day 140, a standardized UV-damage scale (0-12) was lower in mice with ingenol mebutate treatment compared to UVR alone (UVR 10.25 Vs. UVR + ingenol mebutate 6.0; $p=0.002$ ). A topical usage of clobetasol propionate to reduce local skin reactions was also assessed. Surprisingly, clobetasol propionate increased LSR ( $\max$ LSR Tx 1-5: UVR + ingenol mebutate + Clobetasol propionate 3.6-5.5 vs. UVR + ingenol mebutate $2.6-4.3$ ) but at the same time provided better prevention of the photodamage [20].

The treatment with the ingenol mebutate gel could also be beneficial from a social point of view. In Greece, ingenol mebutate $0.05 \%$ and $0.015 \%$ were the most cost-effective topical treatment options, compared to diclofenac and imiquimod (incremental cost-effectiveness ratio of $€ 30,000$ and $€ 10,868$ per quality adjusted lifeyear QALY, respectively) [21].

\section{Conclusions}

To sum up, in our study, the $0.015 \%$ ingenol mebutate gel applied to the face or scalp once a day on 3 consecutive days was effective in treating actinic keratosis.

The big advantage of using ingenol mebutate is that we can achieve a big efficacy after only 2 or 3 applications. Many patients have problems with regular use which can lower effectiveness reported beforehand in clinical trials. A short-time treatment (like 2-3 applications) increases the probability of the patient compliance. Another benefit of a short-time usage is that local reactions disappear relatively quickly. On the face or scalp the peak of the local skin response was observed on day 4 but already on day 15, almost all of it has been resolved.

Our study and all reviewed studies show that ingenol mebutate is highly efficacious and has a generally temporary and mild to moderate local skin response. Even in cases where severe skin reactions appear, the final cosmetic effect is very good and satisfying to our patients. These findings suggest that ingenol mebutate could be a first-line field treatment for actinic keratosis.

\section{Acknowledgments}

The study was funded by Medical University of Lodz, project no. 503/5-064-01/503-01 and 503/1-152-01/503-01.

\section{Conflict of interest}

The authors declare no conflict of interest.

\section{References}

1. Drake LA, Ceilley RI, Cornelison RL, et al. Guidelines of care for actinic keratoses. Committee on Guidelines of Care. J Am Acad Dermatol 1995; 32: 95-8.

2. Bouwes Bavinck JN, Euvrard S, Naldi L, et al. EPI-HPV-UVCA group. Keratotic skin lesions and other risk factors are associated with skin cancer in organ-transplant recipients: a case-control study in the Netherlands, United Kingdom, 
Germany, France, and Italy. I Invest Dermatol 2007; 127: 1647-56.

3. de Berker D, McGregor JM, Hughes BR. British Association of Dermatologists Therapy Guidelines and Audit Subcommittee, Guidelines for the management of actinic keratoses. Br J Dermatol 2007; 156: 222-30.

4. Smith ES, Feldman SR, Fleischer AB, et al. Characteristics of office-based visits for skin cancer. Dermatologists have more experience than other physicians in managing malignant and premalignant skin conditions. Dermatol Surg 1998; 24: 981-5.

5. Halpern AC, Hanson LJ. Awareness of, knowledge of and attitudes to nonmelanoma skin cancer (NMSC) and actinic keratosis (AK) among physicians. Int I Dermatol 2004; 43: 638-42.

6. Krawtchenko N, Roewert-Huber J, Ulrich M, et al. A randomised study of topical $5 \%$ imiquimod vs. topical 5-fluorouracil vs. cryosurgery in immunocompetent patients with actinic keratoses: a comparison of clinical and histological outcomes including 1-year follow-up. Br J Dermatol 2007; 157 Suppl 2: 34-40.

7. Lebwohl M, Swanson N, Anderson LL, et al. Berman, Ingenol mebutate gel for actinic keratosis. N Engl J Med 2012; 366: 1010-9.

8. Spencer JM, Hazan C, Hsiung SH, Robins P. Therapeutic decision making in the therapy of actinic keratoses. J Drugs Dermatol 2005; 4: 296-301.

9. Berlin JM, Rigel DS. Diclofenac sodium 3\% gel in the treatment of actinic keratoses postcryosurgery. J Drugs Dermatol 2008; 7: 669-73.

10. Jorizzo J, Weiss J, Vamvakias G. One-week treatment with $0.5 \%$ fluorouracil cream prior to cryosurgery in patients with actinic keratoses: a double-blind, vehicle-controlled, longterm study. J Drugs Dermatol 2006; 5: 133-9.

11. Jorizzo JL, Markowitz O, Lebwohl MG, et al. A randomized, double-blinded, placebo-controlled, multicenter, efficacy and safety study of 3.75\% imiquimod cream following cryosurgery for the treatment of actinic keratoses. J Drugs Dermatol 2010; 9: 1101-8.

12. Green AC, Beardmore GL. Home treatment of skin cancer and solar keratoses. Australas I Dermatol 1988; 29: 127-30.

13. Ogbourne SM, Suhrbier A, Jones B, et al. Antitumor activity of 3-ingenyl angelate: plasma membrane and mitochondrial disruption and necrotic cell death. Cancer Res 2004; 64: 2833-9.

14. Challacombe JM, Suhrbier A, Parsons PG, et al. Neutrophils are a key component of the antitumor efficacy of topical chemotherapy with ingenol-3-angelate. I Immunol 2006; 177: 8123-32.

15. Jim On S, Knudsen KM, Skov T, Lebwohl M, Regression analysis of local skin reactions to predict clearance of actinic keratosis on the face in patients treated with ingenol mebutate gel: experience from randomized controlled trials. J Drugs Dermatol 2017; 16: 112-4.

16. Loven K, Stein L, Furst K, Levy S. Evaluation of the efficacy and tolerability of $0.5 \%$ fluorouracil cream and $5 \%$ fluorouracil cream applied to each side of the face in patients with actinic keratosis. Clin Ther 2002; 24: 990-1000.

17. Swanson N, Abramovits W, Berman B, et al. Imiquimod $2.5 \%$ and $3.75 \%$ for the treatment of actinic keratoses: results of two placebo-controlled studies of daily application to the face and balding scalp for two 2-week cycles. J Am Acad Dermatol 2010; 62: 582-90.
18. Bucko AD, Jarratt M, Stough DB, et al. Pharmacokinetics of ingenol mebutate gel under maximum use conditions in large treatment areas. J Dermatolog Treat 2017; 29: 74-9.

19. Hashim PW, Nia JK, Singer S, Goldenberg G. An investigatorinitiated study to assess the safety and efficacy of ingenol mebutate $0.05 \%$ gel when used after cryosurgery in the treatment of hypertrophic actinic keratosis on dorsal hands. J Clin Anesthet Dermatol 2016; 9: 16-22.

20. Erlendsson AM, Thaysen-Petersen D, Bay C, et al. Repeated treatments with ingenol mebutate prevents progression of uv-induced photodamage in hairless mice. PLoS One 2016; 11: e0162597.

21. Athanasakis K, Boubouchairopoulou N, Tarantilis F, et al. Cost-effectiveness of ingenol mebutate gel for the treatment of actinic keratosis in Greece. Clin Ther 2017; 39: 993-1002. 